\title{
Effect of software manipulation (Photoshop) of digitised retinal images on the grading of diabetic retinopathy
}

\author{
L D George, J Lusty, D R Owens, R L Ollerton
}

\begin{abstract}
Aims-To determine whether software processing of digitised retinal images using a "sharpen" filter improves the ability to grade diabetic retinopathy.

Methods-150 macula centred retinal images were taken as $35 \mathrm{~mm}$ colour transparencies representing a spectrum of diabetic retinopathy, digitised, and graded in random order before and after the application of a sharpen filter (Adobe Photoshop). Digital enhancement of contrast and brightness was performed and a $\mathrm{X} 2$ digital zoom was utilised. The grades from the unenhanced and enhanced digitised images were compared with the same retinal fields viewed as slides.

Results-Overall agreement in retinopathy grade from the digitised images improved from $83.3 \%$ (125/150) to $94.0 \%$ (141/150) with sight threatening diabetic retinopathy (STDR) correctly identified in $95.5 \%(84 / 88)$ and $98.9 \%(87 / 88)$ of cases when using unenhanced and enhanced images respectively. In total, five images were overgraded and four undergraded from the enhanced images compared with 17 and eight images respectively when using unenhanced images.

Conclusion-This study demonstrates that the already good agreement in grading performance can be further improved by software manipulation or processing of digitised retinal images.

(Br F Ophthalmol 1999;83:911-913)
\end{abstract}

The addition of retinal photography to ophthalmoscopy has been shown to improve sensitivity when grading diabetic retinopathy (DR). ${ }^{1}$ Recently the performance of dedicated digital retinal cameras has been evaluated in a clinical setting when grading DR. ${ }^{2-5}$ We have shown good agreement when grading DR from both digitised colour transparencies (83.3\% (125/ 150) overall agreement) ${ }^{3}$ and digital retinal images taken with the Canon CR5 retinal camera $(93.3 \%(70 / 75) \text { overall agreement })^{2}$ when compared with the same retinal $35 \mathrm{~mm}$ colour transparencies viewed as slides. The digitised colour transparencies in the above study were viewed without software enhancement while the digital images from the Canon CR5 retinal camera were enhanced using the software provided with the camera system (Retinal Imaging Software). Sharpen filters are widely available in commercial image processing software (such as Photoshop, Adobe) and can improve the definition of fine retinal detail which may aid in the grading of DR. We investigated whether software manipulation of digitised images, such as optimisation of contrast and brightness, the application of a sharpen filter, and the use of a X2 digital zoom improved agreement in retinopathy grade between digitised images and $35 \mathrm{~mm}$ colour transparencies.

\section{Materials and methods}

We took 150 macula centred retinal images as $35 \mathrm{~mm}$ colour transparencies and digitised them in Kodak Photo CD format onto CD ROM. The digitised images were anonymised and imported into the commercial image processing package Photoshop (Adobe) at a resolution of $768 \times 512$ pixels. This resolution was chosen so as to be as close to that of currently available digital retinal cameras as possible. The retinal images were graded twice each in separate sessions by a diabetologist with 2 years' grading experience (once without software enhancement and once with software enhancement) in random order in a darkened room. Although not investigated in this study the intraobserver variation of the grader has previously been documented at $6.7 \% .^{2}$ Grading of digitised images was performed on a Sony Triniton 17 inch monitor with contrast and brightness standardised. Grading of the 35 $\mathrm{mm}$ colour transparencies was performed on a Slidex viewer which projects an $11 \times$ magnified image onto a self contained screen. The clinical grading system employed was the Welsh Community Diabetic Retinopathy Study (WCDRS) protocol which is based on the validated European guidelines ${ }^{6}$ (Table 1).

A sharpen filter was applied to all the images graded with software enhancement using the default settings in Photoshop. Contrast and brightness were also enhanced from within the software of Photoshop at the discretion of the grader and a digital zoom facility $(\times 2)$ was used to scrutinise the retina. The original $35 \mathrm{~mm}$ colour transparencies were also anonymised and graded in random order. The grades from the $35 \mathrm{~mm}$ colour transparencies act as the reference against which the grades from the unenhanced and enhanced digitised images are compared. The results are shown below.

\section{Results}

The grades from the unenhanced digitised images are shown compared with the $35 \mathrm{~mm}$ colour transparencies in Table 2 . The grades from the software enhanced digitised images are shown in comparison with the original 35
Accepted for publication 29 March 1999 

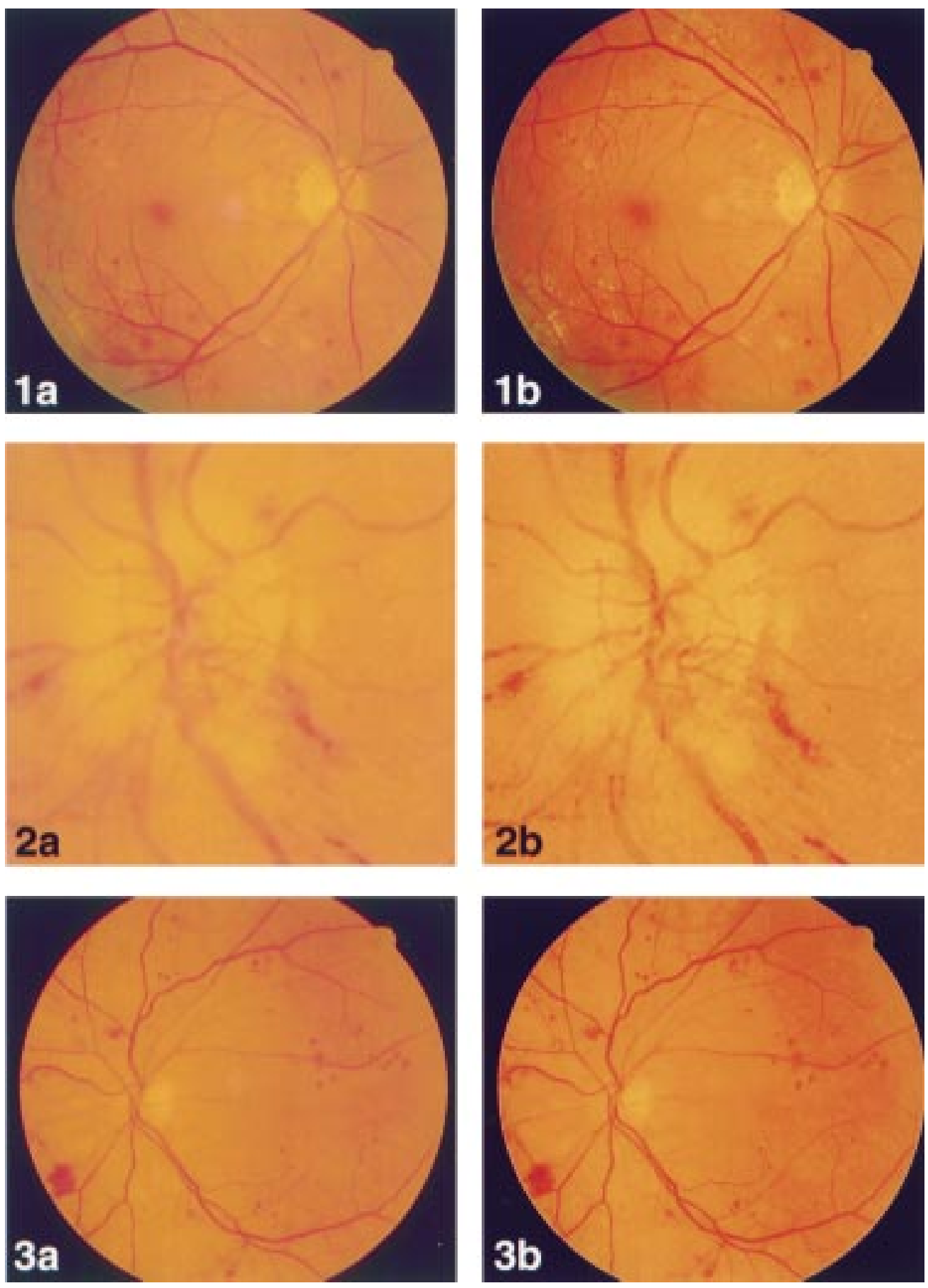

Figures 1-3 Digitised retinal images viewed from within Photoshop. Images 1a, 2a, and $3 a$ have not been digitally enhanced while images $1 b, 2 b$, and $3 b$ have been software enhanced.

mm colour transparencies in Table 3. The shaded diagonal represent exact agreement between the two grading methods with numbers above the line signifying relative undergrading from the digitised images in comparison with the colour transparencies and numbers below the line signifying overgrading.

From the above data it can be seen that the level of exact agreement in retinopathy grades between software enhanced digitised images and the colour transparencies is $94.0 \%(141 /$ 150 ; $95 \%$ CI $87.2-96.3 \%)$ with $100 \%$ (62/62; $95 \%$ CI $94.2-100 \%)$ of non-sight threatening DR (NSTDR) correctly identified and $98.9 \%$
(87/88; 95\%CI 93.8-99.9\%) of sight threatening DR (STDR) correctly identified. Before the images were enhanced the percentage of exact agreement, NSTDR, and STDR correctly identified were $83.3 \%(125 / 150 ; 95 \%$ CI $76.4-88.9 \%), \quad 100 \% \quad(62 / 62 ; 95 \%$ CI $94.2-$ $100 \%)$, and $95.5 \%$ (84/88; 95\%CI $88.8-$ $98.8 \%)$ respectively. In total, five images were overgraded and four images undergraded when using software enhanced images but in only one case did this involve STDR being graded as NSTDR. In comparison, 17 images were overgraded from the unenhanced digitised images and eight were undergraded, four cases 
Table 1 Welsh Community Diabetic Retinopathy Study. (Abbreviated clinical groupings)

Clinical grouping:

0 No diabetic retinopathy.

1 Non-proliferative retinopathy: non-PDR (mild). Occasional haemorrhages and/or microaneurysms and exudates not within one disc diameter of the macula centre. One cotton wool spot per eye not associated with preproliferative lesions.

2a Non-proliferative retinopathy: non-PDR (moderate) without macular involvement. Large circinate or plaque of exudates within the temporal vascular arcades, but not within one disc diameter of the macula centre (fovea).

2 b Non-proliferative retinopathy: non-PDR (moderate) with macular involvement (maculopathy). Haemorrhages and/or exudates within one disc diameter of the macula centre not including microaneurysms.

3 Pre-proliferative retinopathy: (PPDR). Venous irregularities (beading, reduplication, loops) and/or multiple haemorrhages and/or multiple cotton wool spots and/or intraretinal microvascular abnormalities.

4 Proliferative retinopathy: (PDR). New vessels on the disc or elsewhere on the retina Preretinal haemorrhage and/or fibrous tissue.

5 Advanced diabetic eye disease. Vitreous haemorrhage and/or fibrous tissue and/or recent retinal detachment and/or rubeosis iridis.

Note: Diabetic retinopathy grades 1 and 2a regarded as non-sight threatening.

Diabetic retinopathy grades $2 \mathrm{~b}$ and above regarded as sight threatening.

Table 2 Retinopathy grades; unenhanced digitised images versus $35 \mathrm{~mm}$ colour transparencies

\begin{tabular}{llrrlrlllr}
\hline \multicolumn{10}{c}{35 mm colour transparencies } \\
\hline Unenhanced & Grade & 0 & 1 & $2 \mathrm{a}$ & $2 \mathrm{~b}$ & 3 & 4 & 5 & Total \\
digitised & 0 & 7 & 1 & & & & & & 85 \\
images & 1 & 10 & 32 & 1 & 2 & & & & 45 \\
& $2 \mathrm{a}$ & & 4 & 7 & 1 & 1 & & & 13 \\
& $2 \mathrm{~b}$ & & & & 43 & & 1 & & 44 \\
& 3 & & & & 2 & 20 & 1 & & 23 \\
4 & & & & 1 & & 9 & & 10 \\
& 5 & & & & & & & 7 & 7 \\
& Total & 17 & 37 & 8 & 49 & 21 & 11 & 7 & 150 \\
\hline
\end{tabular}

Table 3 Retinopathy grades; software enhanced digitised images versus original $35 \mathrm{~mm}$ colour transparencies

\begin{tabular}{llrrrrrrrr}
\hline \multicolumn{10}{c}{35 mm colour transparencies } \\
\hline $\begin{array}{l}\text { Enhanced } \\
\text { digitised }\end{array}$ & Grade & 0 & 1 & $2 \mathrm{a}$ & $2 \mathrm{~b}$ & 3 & 4 & 5 & Total \\
images & 14 & & & & & & & 14 \\
& 1 & 3 & 36 & 1 & & & & & 40 \\
& $2 \mathrm{a}$ & & 1 & 7 & 1 & & & & 9 \\
& $2 \mathrm{~b}$ & & & & 47 & 1 & & & 48 \\
3 & & & & 1 & 20 & 1 & & 22 \\
4 & & & & & & 10 & & 10 \\
& & & & & & & & 7 & 7 \\
& Total & 17 & 37 & 8 & 49 & 21 & 11 & 7 & 150 \\
\hline
\end{tabular}

of STDR were misgraded from the unenhanced images compared with one case from the enhanced images.

\section{Discussion}

The effects of applying a sharpen filter to retinal images are clearly seen in Figures 1-3 where Figures 1a, 2a, 3a represent the image before sharpening and Figures $1 b, 2 b, 3 b$ the same image after sharpening.

The data show that the already good agreement between retinopathy grades from digitised images and colour transparencies is further improved after software manipulation of the images. Photoshop was chosen for this study as it represents one of the most widely used software packages for image manipulation. Although it is possible to customise the variables around which a sharpen filter works it was felt that for simplicity the default settings should be used, although a more refined approach may improve the results even further.

It was noted that the enhanced images were generally easier to view and grade than the unenhanced images. In particular, the grading of fine retinal detail such as small microaneurysms was consistently more accurate. This can be seen in Table 2 where only three cases of grade 0 retinopathy (from the colour transparencies) were overgraded as grade 1 after manipulation compared with 10 cases before manipulation. There is also generally less scatter of the grades around the shaded diagonal (indicating better agreement) particularly noticeable at the lower end of the spectrum of severity of DR. There were three cases of STDR misgraded only from the unenhanced images and one case misgraded from both the unenhanced and the enhanced images. Of these three cases misgraded before enhancement, two showed small numbers of exudates close to the fovea but these were masked by light reflections from the retina in young patients. The third case showed small numbers of intraretinal microvascular abnormalities which were only visible after enhancement in a retina without other preproliferative features.

We can clearly see that even at the relatively low image resolution utilised in this study the agreement in grading from a digitised image can be improved simply by applying the readily available filters described above, although overenthusiastic use of these filters may result in pixelation or "blockiness" of the images and may even accentuate artefacts in the image.

\section{Conclusion}

Software manipulation of digitised retinal images is associated with an improvement in agreement when grading any DR and also STDR in comparison with the original $35 \mathrm{~mm}$ colour transparencies.

1 Harding SP, Broadbent DM, Neoh C, et al. Sensitivity and specificity of photography and direct ophthalmoscopy in screening for sight threatening eye disease: the Liverpool diabetic eye study. BMF 1995;311:1135-5.

2 George LD, Halliwell M, Hill R, et al. A comparrison of digital retinal images and $35 \mathrm{~mm}$ colour transparencies in detecting and grading diabetic retinopathy. Diabet Med 1998;15:250-3.

3 George LD, Leverton C, Young S, et al. Can digitised colour $35 \mathrm{~mm}$ transparencies be used to diagnose diabetic retinopathy? Diabet Med 1997; 14:970-3.

4 Taylor DJ, Jacob J, Tooke JE. Effectiveness of two commercially available digital retinal screening cameras compared to a Polaroid based system. Diabet Med 1998;15(Suppl ):S63

5 Ryder REJ, Kong N, Bates AS, et al. Instant electronic imaging systems are superior to Polaroid at detecting sight threatening diabetic retinopathy. Diabet Med 1998;15:2548.

6 Retinopathy Working Party. A protocol for screening for diabetic retinopathy in Europe. Diabet Med 1991;8:263-7. 